\title{
Infant swimming in chlorinated pools and the risks of bronchiolitis, asthma and allergy
}

\author{
C. Voisin*, A. Sardella*, F. Marcucci ${ }^{\#}$ and A. Bernard*
}

\begin{abstract}
Recent studies suggest that swimming in chlorinated pools during infancy may increase the risks of lower respiratory tract infection. The aim of the present study was to assess the influence of swimming in chlorinated pools on the risks of bronchiolitis and its late consequences.
\end{abstract}

A total of $\mathbf{4 3 0}$ children (47\% female; mean age 5.7 yrs) in 30 kindergartens were examined. Parents completed a questionnaire regarding the child's health history, swimming practice and potential confounders.

Attendance at indoor or outdoor chlorinated pools ever before the age of 2 yrs was associated with an increased risk of bronchiolitis (OR 1.68; 95\% $\mathrm{Cl} 1.08-2.68 ; \mathrm{p}=0.03$ ), which was exposuredependent for both types of pool ( $p$-value for trend $<0.01$ ). Associations persisted, and were even strengthened, by the exclusion of other risk factors. Among children with no parental antecedents of atopic disease or no day-care attendance, odds ratios for bronchiolitis amounted to 4.45 $(1.82-10.9 ; p=0.001)$ and $4.44(1.88-10.5 ; p=0.007)$ after $>20 \mathrm{~h}$ spent in chlorinated pools during infancy. Infant swimmers who developed bronchiolitis also showed higher risks of asthma and respiratory allergies later in childhood.

Swimming pool attendance during infancy is associated with a higher risk of bronchiolitis, with ensuing increased risks of asthma and allergic sensitisation.

KEYWORDS: Bronchiolitis, infant swimming, respiratory infection, swimming pool

B ronchiolitis is an acute infection of the small airways that primarily affects young infants, most often those aged 224 months. The main causative agent is respiratory syncytial virus (RSV), although other viruses may sometimes be involved. The disease occurs with a seasonal pattern, peaking during winter in temperate climates and the rainy season in tropical climates $[1,2]$. The disease burden of bronchiolitis is substantial and seems to have increased in most developed countries since the 1960s-1970s [3]. In the USA and Europe, the annual incidence of bronchiolitis during the first year of life is estimated to be $10-20 \%$, but incidences as high as 30\% have been reported in some urban areas [3, 4]. Since bronchiolitis increases the risk of childhood asthma $[5,6]$, it also contributes to the rising incidence of chronic respiratory diseases in children.

Factors that may increase the frequency or severity of bronchiolitis are male sex, low gestational age, young or unmarried mothers, infant condition at birth (Apgar score), lack of breastfeeding, early weaning, congenital heart or lung diseases, and a family history of atopic diseases. The risk of bronchiolitis is also higher for infants living with older siblings, attending a day-care centre or exposed to environmental tobacco smoke $[2,7]$. Since ambient air pollution appears to play, if any, a marginal role [8], factors driving the rise of the disease in industrialised countries remain largely unknown.

Infant swimming is a practice that has been greatly popularised in most industrialised countries. NYSTAD and co-workers [9, 10] were among the first to draw attention to the risks that this practice may pose to the airways of infants. These authors noted that infants of atopic parents were more likely to develop wheezing or lower respiratory tract infections when they were attending swimming pools. In a cross-sectional study of schoolchildren aged 10-13 yrs, it was also found that infant swimmers were more likely to develop asthma and recurrent bronchitis than their peers who had never attended a chlorinated pool before the age of 2 yrs [11, 12]. Interestingly, this poorer respiratory health of infant swimmers was associated with lower serum levels of Clara cell protein (CC16), a protein protecting from inflammation in acute RSV infection [13]. These findings led to the postulate that the chlorine used to disinfect swimming pools can cause airway
AFFILIATIONS

*Dept of Public Health, Catholic University of Louvain, Louvain, Belgium, and

\#Dept of Pediatrics, University Dept of Medical and Surgical Specialties and Public Health, Perugia, Italy.

CORRESPONDENCE

A. Bernard

Faculty of Medicine Catholic University of Louvain Avenue E. Mounier 53.02 B-1200 Brussels Belgium

E-mail: Alfred.bernard@uclouvain.be

Received:

July 232009

Accepted after revision: Dec 302009

First published online: Jan 142010 
changes making the lungs more sensitive to infection- and asthma-inducing agents [12]. A Swedish study also described decreased levels of serum CC16 in children who swam during infancy compared to those who did not [14], providing further evidence that terminal airways and, in particular, Clara cells may be damaged during infant swimming.

Given these associations between infant swimming, serum CC16 and RSV infection, it was hypothesised that the infant swimming practice should be associated with a higher risk of bronchiolitis. In order to test this hypothesis, the associations between infant swimming, bronchiolitis and its sequelae were explored in a cross-sectional study focused on young schoolchildren. Since both outdoor and indoor pools may affect the airways of swimmers [15], the present study was not limited to baby swimming lessons in indoor pools, but considered all kinds of swimming activity during infancy, regardless of the type of chlorinated pool and the conditions of attendance.

\section{METHODS}

\section{Study population}

The study participants were 5-6-yr-old children from 30 schools located mainly in the areas of Brussels and Liège (Belgium). These children were recruited in the framework of a prospective study on the respiratory impact of air pollution. A questionnaire and an informed consent document were distributed to children in the third year of kindergarten. Of the 839 children who received these documents, 430 returned the questionnaire with the informed consent document filled in by the parents. The overall participation rate was $51.3 \%$, but showed great variation between schools (20-90\%). Since the participation rate did not correlate with the prevalence of the main outcomes and risk factors, it was decided to retain all schools for the statistical analysis and to add the participation rate to the list of potential confounders. The ethics committee of the Faculty of Medicine of the Catholic University of Louvain (Louvain, Belgium) approved the study protocol, which complied with all applicable requirements of the international regulations.

\section{Protocol}

Parents completed a detailed questionnaire inquiring about the health of their child, respiratory symptoms, family antecedents, care during early life and all lifestyle or environmental factors known or suspected to influence the risk of bronchiolitis. Questions regarding respiratory symptoms were those of the International Study of Asthma and Allergies in Childhood [16]. Wheezing was identified by a positive answer to the question "Has your child had episodes of wheezing during the last 12 months?". The questionnaire included specific questions asking whether the child had ever been diagnosed for most common childhood diseases. Among these, there were seven distinct questions about the following respiratory diseases: asthma, bronchitis, bronchiolitis, pneumonia (or bronchopneumonia), hay fever, allergic rhinitis, and sinusitis. For each question, the parents were asked to reply "yes" or "no". In the case of a positive reply, parents were then asked to indicate the exact age at which the disease was diagnosed. The questionnaire also comprised questions about sport and recreational activities. For swimming practice, parents were asked to specify the type of pool attended by their child, the type of disinfection method used (even though almost all of them use chlorine), the frequency of attendance and the age at which their child started to attend the pool regularly. This information served to calculate the cumulative pool attendance (CPA) at indoor or outdoor chlorinated pools, separately or combined, before the age of 2 yrs (during infancy) or later in life (during childhood). Children were examined in schools during 09:00-16:00 h. Height and body weight were measured. Since the examination of children in schools precluded any provocation test, screening for respiratory allergies in school was performed noninvasively by measuring specific immunoglobulin $\mathrm{E}$ in nasal mucosa using the Rhinostick test (made by F. Marcucci) [17]. This test was successfully performed in 372 children, in whom the following allergens were screened for: cat epithelium, Dermatophagoides pteronyssinus, Anthoxanthum odoratum, Parietaria officinalis and a mix of tree allergens containing Betula odorata, Corylus avellana, Carpinus betulus and Alnus incana. Regarding sport and recreational activities organised by the schools, the school directors were asked to complete a questionnaire that permitted the information given by the parents to be cross-checked and completed.

\section{Swimming pools}

In Belgium, every public swimming pool is legally required to regularly check the microbial and chemical quality of the water by measuring several parameters, including active (0.5$1.5 \mathrm{ppm})$ and combined chlorine ( $<2 \mathrm{ppm})$. In 2003, the legislation was strengthened by a lowering of the standard for combined chlorine $(<0.8 \mathrm{ppm})$ and the setting of a standard for trichloramine in pool air $\left(<500 \mu \mathrm{g} \cdot \mathrm{m}^{-3}\right.$ in air sampled $1.5 \mathrm{~m}$ above the pool surface). Since there were no public outdoor pools in the areas studied, the open-air swimming pools attended by infant swimmers were mostly residential. In Belgium, as in most countries, there are no specific regulations for these privately owned swimming pools, which are disinfected according to the instructions of the chlorine supplier (active chlorine 1-2 ppm).

\section{Statistical analysis}

Continuous variables were described using the median with interquartile range. Differences between infant swimmers and their controls were assessed using the two-sided MannWhitney U-test for the CPA indices and an unpaired t-test for the other variables. Binary variables were compared using the Chi-squared test, or with a Chi-squared test for trend for the analysis of exposure-response relationships. Logistic regression models were used to assess the associations between swimming and bronchiolitis while adjusting for potential confounders. CPA at outdoor or indoor pools before the age of 2 yrs was categorised as never, $>0-20 \mathrm{~h}$ and $>20 \mathrm{~h}$. Crude and adjusted odds ratios (ORs) for the outcomes were calculated using as reference level the occurrence of the outcome among children who never attended an indoor or outdoor chlorinated pool before the age of 2 yrs. A backward approach was used, including all potential control variables and removing the least significant predictor until the model only contained variables with a p-value of $<0.20$. A total of 24 potential predictors were tested, including, among others, age, sex, parental asthma and/or respiratory allergies (hay fever or allergic rhinitis), high parental educational level (father and/or mother graduate of university or high school), maternal smoking during pregnancy, parental smoking at home, season 
of birth, birthweight, number of older siblings, house cleaning with bleach, breastfeeding, day-care centre attendance, area of current residence (Liège versus Brussels area), having spent infancy in a urban or rural area, living in the vicinity of a polluting industry or within a distance of $100 \mathrm{~m}$ of a busy road, and cumulative time spent in indoor or outdoor chlorinated swimming pools before the age of 2 yrs, separately or in combination (CPA). A classification of CPA in tertiles was also used. For outcomes other than bronchiolitis, CPA at outdoor or indoor swimming pools after the age of 2 yrs was added to the potential predictors. Statistical analyses were performed using SAS version 9.1.3 (SAS, Cary, NC, USA).

\section{RESULTS}

Table 1 compares the characteristics of infant swimmers in indoor or outdoor chlorinated pools $(n=195)$ and children who never swam before the age of 2 yrs $(n=235)$. On average, infant swimmers had spent $6 \mathrm{~h}$ in indoor and $8 \mathrm{~h}$ in outdoor chlorinated pools before the age of $2 \mathrm{yrs}$. These infant swimmers also showed a greater lifetime CPA at indoor or outdoor chlorinated swimming pools (total indoor and outdoor CPA 132 versus $35 \mathrm{~h}$ in other children) (table 1). There were no significant differences between the two groups regarding most risk factors of respiratory disease, including sex, age, body mass index, birthweight, exposure to tobacco smoke and parental allergies. The social class or socioeconomic status, as evaluated on the basis of parental education, and the proportions of children with parental asthma, who had been breastfed or attended a day-care centre were higher in the group of swimmers than in non-swimmers. The two groups did not differ with respect to the prevalences of wheezing and ever-diagnosed asthma, respiratory allergies, bronchitis, sinusitis and pneumonia. The prevalence of bronchiolitis was, however, significantly greater in infant swimmers than in their controls (36.4 versus $23.8 \%$; $\mathrm{p}=0.004$ ).

The logistic regression analysis confirmed the association between infant swimming and the risk of bronchiolitis (OR $1.68 ; 95 \%$ CI 1.08-2.68; $\mathrm{p}=0.03)$. Other risk factors of bronchiolitis identified included male sex (OR 1.65; 95\% CI 1.052.59; $\mathrm{p}=0.03)$, parental asthma and/or respiratory allergies (OR 1.73; 95\% CI 1.20-2.74; $\mathrm{p}=0.02$ ), number of older siblings (OR 1.69; 95\% CI 1.07-2.67; $\mathrm{p}=0.02$ ), maternal smoking during pregnancy (OR 1.80; 95\% CI 1.01-3.37; p=0.05) and day-care centre attendance (OR 1.87; 95\% CI 1.17-3.00; $\mathrm{p}=0.009$ ). Breastfeeding, by contrast, was associated with a protective effect towards bronchiolitis (OR 0.58; 95\% CI 0.34-0.97; $\mathrm{p}=0.04$ ). The parental educational level, a surrogate of socioeconomic status, the use of bleach for house cleaning or parental smoking at home did not emerge as significant predictors of bronchiolitis ( $p>0.20$ for all).

The analysis was pursued by examining whether or not the risk of bronchiolitis was different when attending indoor or outdoor chlorinated pools. As shown in table 2, the OR for bronchiolitis increased dose-dependently with CPA at both types of swimming pool considered separately ( $p$-value for trend $<0.05$ for both). Stratification of infant swimmers into tertiles of CPA at indoor or outdoor chlorinated pools led to similar patterns of significant increases ( $p$-value for trend 0.009 for indoor and 0.03 for outdoor pools; data not shown). The same pattern of exposure-related increases was found when

\begin{tabular}{|c|c|c|c|}
\hline & $\begin{array}{l}\text { Swimming } \\
\text { infants }\end{array}$ & Controls & p-value \\
\hline Subjects n & 195 & 235 & \\
\hline Males & $105(53.9)$ & $124(52.8)$ & 0.82 \\
\hline School participation rate \% & $54.9 \pm 19.4$ & 58. $3 \pm 23.0$ & 0.19 \\
\hline Age yrs & $5.60 \pm 0.38$ & $5.68 \pm 0.38$ & 0.79 \\
\hline BMI $\mathbf{k g} \cdot \mathrm{m}^{-2}$ & $20.9 \pm 3.8$ & $20.6 \pm 3.5$ & 0.39 \\
\hline \multicolumn{4}{|l|}{ Parents } \\
\hline Higher educational level & $154(79.0)$ & $156(66.4)$ & 0.004 \\
\hline Asthma & $38(19.5)$ & $25(10.6)$ & 0.01 \\
\hline Asthma and/or respiratory allergies & $86(44.1)$ & $88(37.4)$ & 0.16 \\
\hline \multicolumn{4}{|l|}{ Early life } \\
\hline Birthweight g & $3206 \pm 639$ & $3436 \pm 2396$ & 0.30 \\
\hline Breastfeeding & $161(82.6)$ & 169 (71.9) & 0.01 \\
\hline Day-care centre attendance & $127(65.1)$ & $102(43.4)$ & $<0.001$ \\
\hline \multicolumn{4}{|l|}{ Exposure to tobacco smoke } \\
\hline During pregnancy & $31(16.0)$ & $32(13.6)$ & 0.51 \\
\hline Parental smoking at home & $55(28.2)$ & $74(31.5)$ & 0.46 \\
\hline \multicolumn{4}{|l|}{ CPA before age of $2 \mathrm{yrs} h$} \\
\hline Indoor & $6(0-21)$ & & \\
\hline Outdoor & $8(0-31)$ & & \\
\hline Total & $22(8-50)$ & & \\
\hline \multicolumn{4}{|l|}{ CPA over lifetime $h$} \\
\hline Indoor & $53(17-129)$ & $18(0-48)$ & $<0.001$ \\
\hline Outdoor & $50(4-146)$ & $0(0-28)$ & $<0.001$ \\
\hline Total & $132(52-202)$ & $35(5-91)$ & $<0.001$ \\
\hline \multicolumn{4}{|l|}{ Environment and lifestyle } \\
\hline Number of older siblings & $0.87 \pm 0.79$ & $0.92 \pm 1.12$ & 0.66 \\
\hline House cleaning with bleach & $43(22.1)$ & $66(28.1)$ & 0.15 \\
\hline Living $<100 \mathrm{~m}$ from a busy road & $63(32.3)$ & 79 (33.6) & 0.78 \\
\hline Exposure to pets since birth & $85(43.6)$ & $99(42.1)$ & 0.76 \\
\hline \multicolumn{4}{|l|}{ Aeroallergen-specific nasal lg $\mathrm{E}^{\#}$} \\
\hline House dust mite & $21(12.2)$ & $22(11.0)$ & 0.71 \\
\hline Cat & $15(8.7)$ & $14(7.0)$ & 0.54 \\
\hline Pollen & $27(15.7)$ & $27(13.5)$ & 0.55 \\
\hline At least one aeroallergen & $42(24.4)$ & $48(24.0)$ & 0.92 \\
\hline \multicolumn{4}{|l|}{$\begin{array}{l}\text { Respiratory symptoms and } \\
\text { diseases }\end{array}$} \\
\hline Wheezing ${ }^{+}$ & $36(18.5)$ & $40(17.0)$ & 0.70 \\
\hline Asthma & $16(8.2)$ & $15(6.4)$ & 0.47 \\
\hline Hay fever & $17(8.7)$ & $27(11.5)$ & 0.35 \\
\hline Allergic rhinitis & 27 (13.9) & 25 (10.6) & 0.31 \\
\hline Bronchiolitis & $71(36.4)$ & $56(23.8)$ & 0.004 \\
\hline Bronchitis & $93(47.7)$ & $111(47.2)$ & 0.92 \\
\hline Sinusitis & $28(14.4)$ & 30 (12.8) & 0.63 \\
\hline Pneumonia & $20(10.3)$ & 28 (11.9) & 0.59 \\
\hline
\end{tabular}

Data are presented as mean $\pm \mathrm{SD}, \mathrm{n}(\%)$ or median (interquartile range) unless otherwise indicated. BMI: body mass index; CPA: cumulative pool attendance; Ig: immunoglobulin. ${ }^{\#}: 172$ children were tested in the swimming infant group and 200 in the other group; ${ }^{\circ}$ : defined as doctor-diagnosed diseases at any time; ${ }^{+}$: episodes of wheezing during the last 12 months.

the analysis was based on children attending only indoor or only outdoor pools to the exclusion of the other type of pool. 


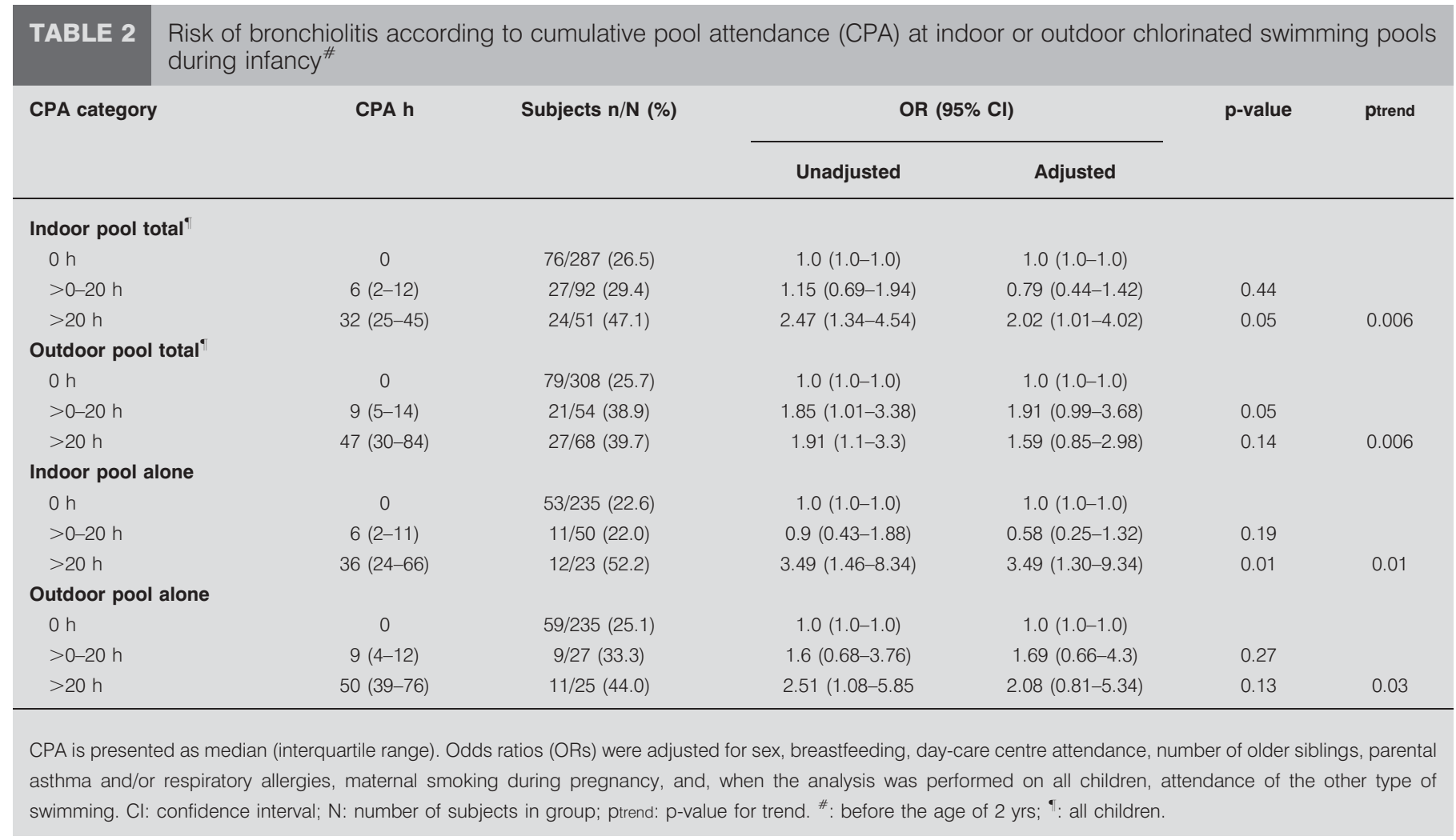

It was also ascertained that these associations were not confounded by differences in participation rate between schools or by other risk factors for bronchiolitis, especially those linked to the socioeconomic status of the children, which, indeed, was higher in infant swimmers than in controls. This analysis was conducted by combining the CPA at indoor and outdoor pools in order to obtain sufficient subjects in the different pool attendance categories. As shown in table 3, associations between bronchiolitis and infant swimming persisted, and were very consistent (OR for CPA of $>20 \mathrm{~h}$ was 2.2-2.7, $\mathrm{p} \leqslant 0.03$ ) across categories created by excluding children from schools with a low participation rate $(<50 \%)$, children from parents with a lower educational level, children who had not been breastfed, children who had been exposed to tobacco smoke or else who had lived with older siblings or in a house cleaned with bleach. Quite interestingly, exclusion of children of parents with asthma or respiratory allergies, or of children having attended a day-care centre, two well-known risk factors for bronchiolitis, noticeably strengthened the associations between bronchiolitis and infant swimming (OR for CPA of $>20 \mathrm{~h} 4.45$ and 4.44; $\mathrm{p}=0.001$ and 0.007, respectively), which emerged through a particularly remarkable dose-response relationship among children who had never attended a day-care centre $(\mathrm{p}<0.001)$.

Lastly, the temporal coherence of these associations was checked by comparing the age of bronchiolitis occurrence with the age at which the child started to attend swimming pools. Of the 71 infant swimmers who had bronchiolitis, there were 54 cases for which the parents provided the exact dates when their child started swimming and developed bronchiolitis. On average, these infant swimmers developed bronchiolitis at the age of $9.6 \pm 5.9$ months and were diagnosed with asthma at the age of $21.8 \pm 18.6$ months. Among these, bronchiolitis occurred after the start of infant swimming in $35(65 \%)$ cases, a number consistent with the excess of bronchiolitis cases observed in the infant swimming group. Children who never swam during infancy had bronchiolitis at about the same age as infant swimmers ( $9.3 \pm 5.5$ months), but were diagnosed with asthma later, at the age $30.6 \pm 10.0$ months.

Since bronchiolitis is known to increase the risk of wheezing, asthma or allergic sensitisation in subsequent years, the late consequences of bronchiolitis were also compared between infant swimmers and nonswimmers. This comparison was made by adjusting the ORs for CPA at indoor or outdoor pools after the age of 2 yrs, which, indeed, was very different between the two groups. Table 4 clearly shows that bronchiolitis was associated with an increased risk of wheezing, doctordiagnosed asthma and hay fever only among children who had attended chlorinated pools during their infancy. Interestingly, bronchiolitis and infant swimming also interacted to increase the risk of sensitisation to house dust mite $(p=0.04)$ and pollen $(p=0.05)$. There were no differences in the ORs for cat allergy and allergic rhinitis between the two groups. When considering children who never developed bronchiolitis, there were no significant differences in the prevalences of respiratory diseases and sensitisation to aeroallergens between children who swam during infancy and those who did not $(\mathrm{p}>0.07$ for all; Chi-squared test) (table 4).

\section{DISCUSSION}

The present study shows that swimming in indoor and outdoor pools during infancy is associated with an exposurerelated increase in the risk of bronchiolitis. This effect is independent of other known risk factors for bronchiolitis, such 


\begin{tabular}{|c|c|c|c|c|c|c|c|c|c|c|c|c|c|}
\hline 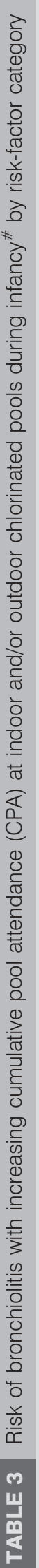 & 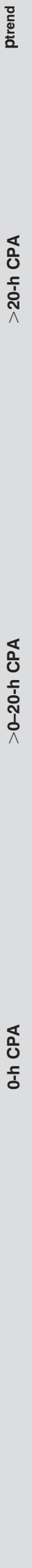 & 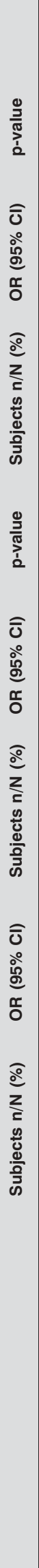 & 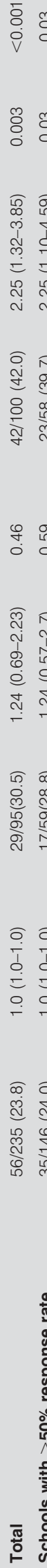 & 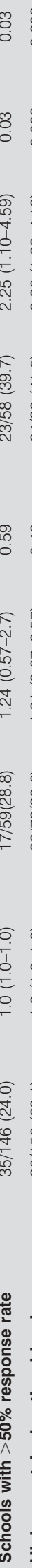 & 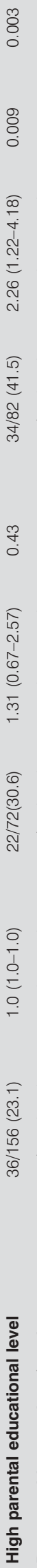 & 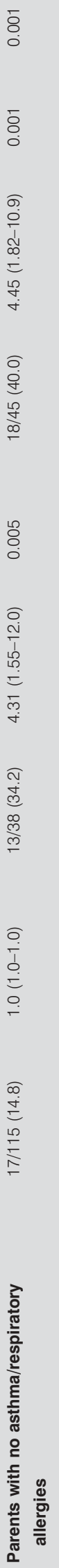 & 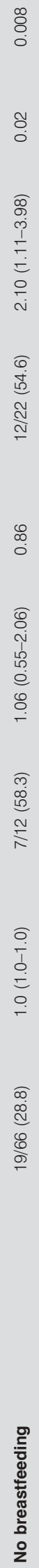 & 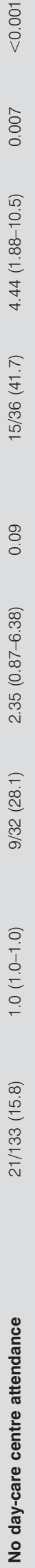 & 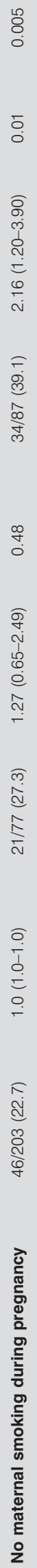 & 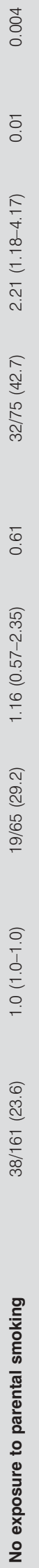 & 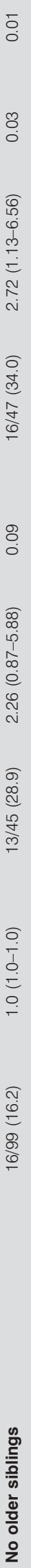 & 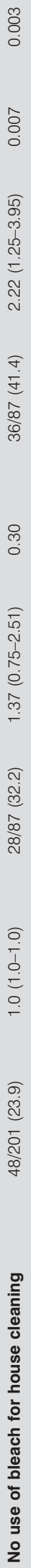 & 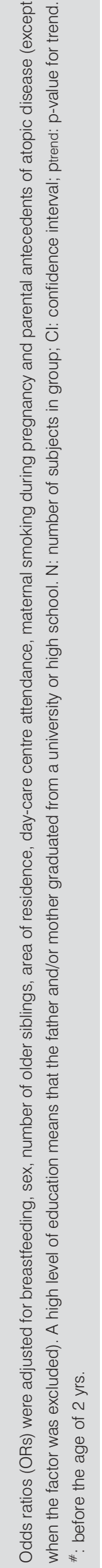 \\
\hline
\end{tabular}

as day-care attendance, exposure to tobacco smoke or parental antecedents of atopic diseases. The present study also shows that, among children who had bronchiolitis, only those who were infant swimmers were at greater risk of asthma and respiratory allergies in subsequent years.

These results are in concordance with the observation of NYSTAD and co-workers $[9,10]$ that swimming before the age of 2 yrs increases the prevalence of lower respiratory tract infection. They are also consistent with a prior study on schoolchildren [11, 12], suggesting that infant swimming may cause airway changes predisposing to asthma and recurrent bronchitis. In the present study focusing on younger children, no significant differences were found between infant swimmers and other children regarding the risk of asthma and allergies. This lack of association between infant swimming and asthma, reported by other authors for children of the same age [18-20], does not necessarily argue against the hypothesis of a causal link between infant swimming and poorer respiratory health later during childhood. Children aged 5-6 yrs are probably too young to detect associations with chronic respiratory diseases that develop and are correctly diagnosed later during childhood. This might explain why studies linking swimming pool attendance to childhood asthma were all based on children with a mean age of $>9$ yrs [21-23]. The follow-up of the present cohort of infant swimmers should provide more conclusive data regarding the long-term consequences of infant swimming.

The swimming pool factor responsible for the risk of bronchiolitis is hard to identify with certainty given the multiplicity of potentially harmful agents in the swimming pool environment [20, 24]. Currently, one of the most plausible explanations is that the airways of infant swimmers are made more sensitive to infections because of the irritating effects of the chlorine used to disinfect swimming pools. The fact that bronchiolitis is increased by outdoor, as well as by indoor, pools means that chloramines are not the sole and probably not the main irritants that might be implicated in the risk of bronchiolitis. The first reason for this is that the outdoor pools attended by infant swimmers in the present study were mainly residential pools. Compared to public pools, residential pools are, indeed, much less polluted by organic matter from bathers, in particular by urine, which is the main source of nitrogen leading to the formation of chloramines. The second reason is that trichloramine, the ultimate chlorination by-product, is a highly volatile gas that is very quickly dispersed into the atmosphere once released at the surface of open-air pools, which, therefore, do not have the characteristic chlorine smell of indoor pools. As for the risks of asthma and respiratory allergies associated with outdoor pools [15], we suspect that the major burden of oxidants irritating the airways of infant swimmers comes from the microaerosols or small volumes of water that they inhale while actively playing and having their head under water [12]. The risk of inhaling small volumes during submersion exercise is especially important since infants cannot control their breathing. If the infant swimming practice is considered to be safe, it is because of the laryngeal or gag reflex (closure of the larynx with the epiglottis) that is triggered when water gets into the infant's mouth. This gag 


\begin{tabular}{|c|c|c|c|c|c|c|c|c|c|c|}
\hline \multirow[t]{4}{*}{ TABLE 4} & $\begin{array}{l}\mathrm{k} \text { of res } \\
\text { ir contrc }\end{array}$ & atory dise & ases and sen & sitisation to ae & roallerg & s assoc & ed with $b$ & onchiolitis in s & vimming infant & s and \\
\hline & \multicolumn{5}{|c|}{ Controls } & \multicolumn{5}{|c|}{ Swimming infants } \\
\hline & \multicolumn{2}{|c|}{ Bronchiolitis n (\%) } & \multicolumn{2}{|c|}{ OR (95\% Cl) } & \multirow[t]{2}{*}{$p$-value } & \multicolumn{2}{|c|}{ Bronchiolitis n (\%) } & \multicolumn{2}{|c|}{ OR (95\% Cl) } & \multirow[t]{2}{*}{ p-value } \\
\hline & No & Yes & Unadjusted & Adjusted & & No & Yes & Unadjusted & Adjusted & \\
\hline Subjects $n$ & 179 & 56 & & & & 124 & 71 & & & \\
\hline Wheezing & $28(15.6)$ & $12(21.4)$ & $1.47(0.69-3.13)$ & $1.29(0.47-3.54)$ & 0.62 & $18(14.5)$ & $18(25.4)$ & $2.00(0.96-4.16)$ & $2.49(1.13-5.49)$ & 0.03 \\
\hline Asthma & $11(6.2)$ & $4(7.1)$ & $1.18(0.36-3.85)$ & $0.91(0.23-3.70)$ & 0.90 & $5(4.0)$ & $11(15.5)$ & $4.36(1.45-13.1)$ & 8.27 (2.30-29.6) & 0.001 \\
\hline Allergic rhinitis & $16(8.9)$ & $9(16.1)$ & $1.95(0.81-4.70)$ & $2.08(0.79-5.40)$ & 0.14 & $13(10.5)$ & 14 (19.7.) & $2.10(0.92-4.76)$ & $4.65(1.42-15.2)$ & 0.01 \\
\hline Hay fever & $19(10.6)$ & $12(21.4)$ & $1.40(0.58-3.41)$ & $0.96(0.34-2.70)$ & 0.94 & $6(4.8)$ & $11(15.5)$ & $3.61(1.27-10.2)$ & $4.70(1.52-14.5)$ & 0.007 \\
\hline \multicolumn{11}{|l|}{ Nasal IgE } \\
\hline Pollen\# & $24(15.7)$ & $3(6.4)$ & $0.39(0.11-1.38)$ & $0.57(0.15-2.10)$ & 0.40 & $14(12.8)$ & $13(20.6)$ & $1.86(0.80-4.30)$ & $2.63(1.01-6.80)$ & 0.05 \\
\hline $\mathrm{HDM}^{\#}$ & $15(9.8)$ & $7(14.9)$ & $1.62(0.62-4.25)$ & $2.05(0.62-6.75)$ & 0.24 & $9(8.3)$ & $21(33.3)$ & $2.59(1.02-6.55)$ & $2.86(1.07-7.80)$ & 0.04 \\
\hline $\mathrm{Cat}^{\#}$ & $11(7.2)$ & $3(6.4)$ & $0.89(0.24-3.32)$ & $0.90(0.20-4.18)$ & 0.90 & $8(7.3)$ & $7(11.1)$ & $1.56(0.54-5.54)$ & $1.26(0.40-3.96)$ & 0.69 \\
\hline
\end{tabular}

Outcomes are defined in table 1. Bronchiolitis was diagnosed at a mean \pm SD age of $9.6 \pm 5.9$ months in infant swimmers and $9.3 \pm 5.5$ months in controls. The prevalences of respiratory diseases and sensitisation to aeroallergens among children who never developed bronchiolitis did not differ significantly between swimming infants and their controls ( $p>0.07$ for all; Chi-squared test). OR: odds ratio; Cl: confidence interval; Ig: immunoglobulin; HDM: house dust mite. ${ }^{*}$ : a total of 153 and 47 controls and 109 and 63 swimming infants, respectively, who did not or did develop bronchiolitis, were tested.

reflex disappears, however, when the infant gets older (>6 months), and, anyway, this reflex cannot prevent small amounts of chlorinated water deposited or trapped in the upper respiratory tract being conducted more deeply into the lungs when the infant surfaces to breathe [12].

The most reactive and concentrated chlorine compound present in the water and microaerosols of both outdoor and indoor pools is hypochlorite/hypochlorous acid, i.e. the active chlorine itself (concentrations ranging 1-2 ppm). Depending on the level of organic pollution of pool water, infant swimmers have also been exposed to chlorination byproducts, among which the most irritating and concentrated are mono-, dichlor- and trichloramine, as well as dichloromethylamine [19, 20, 24, 25]. Since public pools are usually more polluted by nitrogenous substances from bathers (urine, sweat and saliva) than residential pools, irritating effects caused by the chloramines might explain why the ORs for bronchiolitis are higher with indoor than with outdoor pools.

Chlorine-based oxidants, such as hypochlorous acid or chloramines, are known to be potent oxidants capable of damaging the endothelial and epithelial barriers [26, 27]. Studies based on lung injury markers have shown that, at concentrations commonly found in indoor pools, these chemicals can affect the permeability or the cellular integrity of the deep lung epithelium. Regular attendance at chlorinated pools by schoolchildren has been associated with an exposuredependent increase in lung epithelium permeability (lung hyperpermeability), resulting in intravascular leakage of surfactant-associated proteins A and B [28]. A decrease in serum CC16 has also been described in children who use indoor pools during their infancy or later in childhood, which is a reflection of a decrease in Clara cell numbers and an ensuing decrease in CC16 production [11, 12, 14]. The latter observation is particularly relevant to the present study given the experimental evidence that CC16 downregulates inflammation during acute RSV infection [13].

Even though airways irritation by chlorine-based oxidants appears to be a very likely explanation for the increased risk of bronchiolitis associated with infant swimming, other causative factors might also play a role. As a result of inadequate disinfection with chlorine, the swimming pool environment can be contaminated by viruses (adeno-, noroand echovirus), which may cause outbreaks of water-borne illnesses, such as gastroenteritis, dermatitis and respiratory infections [29]. Respiratory illnesses in these incidents are rather uncommon, and, in most cases, limited to the upper airways. Nevertheless, we have no evidence permitting us to exclude the possibility that the swimming pool environment may increase the risks of cross-infection with $\operatorname{RSV}[30,31]$. Another possibility that should be considered is the inhalation of swimming pool water when infants perform submersion activities. Owing to its hypotonicity, swimming pool water deposited in airways might, perhaps, cause some airway alterations, aggravating the toxic effects of chlorination products [32].

The present study has certain limitations. The most important one concerns exposure assessment, since it was obviously not possible to retrieve exposure data regarding the levels of chlorine in the indoor or outdoor swimming pools that the children attended during infancy. There was no choice but to use the information provided by the parents. The existence of exposure-response relationships for both outdoor and indoor pools suggests that the lack of data regarding the precise levels of chlorine in swimming pools has not been critical to the point of distorting the present analysis. Another limitation concerns the participation rate, which only reached $51 \%$ overall and varied greatly between schools. The young age of the children 
combined with the fact that the medical examination, though based on noninvasive tests, was performed in schools probably deterred a significant proportion of parents from participating in the study. This low participation rate does not seem to have biased the study since there were no significant correlations between participation rate and the prevalences of the main outcomes and risk factors. The response rate, tested along with other potential confounders, did not emerge as a significant predictor of bronchiolitis either. This lack of confounding by the participation rate probably stems from the fact that parents were blinded to the tested hypothesis. Since the study was conducted in the framework of a prospective study on environmental factors affecting the respiratory health of children, infant swimming was only one of the many items addressed by the questionnaire.

In summary, the present study shows that infant swimming is associated with a dose-dependent increase in the risk of bronchiolitis. Exposure to chlorinated pools during infancy also interacts with bronchiolitis to increase the risks of asthma and respiratory allergies later during childhood, which suggests that the infant swimming practice may have a more long-standing impact on the respiratory health of children.

\section{SUPPORT STATEMENT}

This study was supported by the Belgian Science Policy (Brussels, Belgium) (ANIMO (Indoor risk factors for childhood respiratory diseases: development and application of non-invasive biomarkers) project). A. Bernard is Research Director of the National Fund for Scientific Research (Brussels, Belgium).

\section{STATEMENT OF INTEREST}

None declared.

\section{REFERENCES}

1 American Academy of Pediatrics Subcommittee on Diagnosis and Management of Bronchiolitis.: Diagnosis and management of bronchiolitis. Pediatrics 2006; 118: 1774-1793.

2 Smyth RL, Openshaw PJ. Bronchiolitis. Lancet 2006; 368: 312-322.

3 Carroll KN, Gebretsadik T, Griffin MR, et al. Increasing burden and risk factors for bronchiolitis-related medical visits in infants enrolled in a state health care insurance plan. Pediatrics 2008; 122: 58-64.

4 Grimpel E. Epidémiologie de la bronchiolite du nourrison en France. [Epidemiology of infant bronchiolitis in France.] Arch Pediatr 2001; 8: 83S-92S.

5 Lemanske RF Jr, Jackson DJ, Gangnon RE, et al. Rhinovirus illnesses during infancy predict subsequent childhood wheezing. J Allergy Clin Immunol 2005; 116: 571-577.

6 Cassimos DC, Tsalkidis A, Tripsianis GA, et al. Asthma, lung function and sensitization in school children with a history of bronchiolitis. Pediatr Int 2008; 50: 51-56.

7 Koehoorn M, Karr CJ, Demers PA, et al. Descriptive epidemiological features of bronchiolitis in a population-based cohort. Pediatrics 2008; 122: 1196-1203.

8 Karr CJ, Rudra CB, Miller KA, et al. Infant exposure to fine particulate matter and traffic and risk of hospitalization for RSV bronchiolitis in a region with lower ambient air pollution. Environ Res 2009; 109: 321-327.

9 Nystad W, Nia F, Magnus P, et al. Baby swimming increases the risk of recurrent respiratory tract infections and otitis media. Acta Paediatr 2003; 92: 905-909.

10 Nystad W, Håberg SE, London SJ, et al. Baby swimming and respiratory health. Acta Paediatr 2008; 97: 657-662.
11 Bernard A, Carbonnelle S, Nickmilder M, et al. Non-invasive biomarkers of pulmonary damage and inflammation: application to children exposed to ozone and trichloramine. Toxicol Appl Pharmacol 2005; 206: 185-190.

12 Bernard A, Carbonnelle S, Dumont $X$, et al. Infant swimming practice, pulmonary epithelium integrity, and the risk of allergic and respiratory diseases later in childhood. Pediatrics 2007; 119: 1095-1103.

13 Wang $\mathrm{S}$, Rosenberger $\mathrm{C}$, Bao $\mathrm{Y}$, et al. Clara cell secretory protein modulates lung inflammatory and immune responses to respiratory syncytial virus infection. J Immunol 2003; 171: 1051-1060.

14 Lagerkvist BJ, Bernard A, Blomberg A, et al. Pulmonary epithelial integrity in children: relationship to ambient ozone exposure and swimming pool attendance. Environ Health Perspect 2004; 112: 1768-1771.

15 Bernard A, Nickmilder M, Voisin C. Outdoor swimming pools and the risks of asthma and allergies during adolescence. Eur Respir J 2008; 32: 979-988.

16 Beasley $\mathrm{R}$, the International Study of Asthma and Allergies in Childhood (ISAAC) Steering Committee, Worldwide variation in prevalence of symptoms of asthma, allergic rhinoconjunctivitis, and atopic eczema: ISAAC. Lancet 1998; 351: 1225-1232.

17 Marcucci F, Sensi L. A new method for IgE detection in nasal mucosa. Clin Exp Allergy 1989; 19: 157-162.

18 Schoefer Y, Zutavern A, Brockow I, et al. Health risks of early swimming pool attendance. Int J Hyg Environ Health 2008; 211 367-373.

19 Uyan ZS, Carraro S, Piacentini G, et al. Swimming pool, respiratory health, and childhood asthma: should we change our beliefs? Pediatr Pulmonol 2009; 44: 31-37.

20 Weisel CP, Richardson SD, Nemery B, et al. Childhood asthma and environmental exposures at swimming pools: state of the science and research recommendations. Environ Health Perspect 2009; 117: 500-507.

21 Bernard A, Carbonnelle S, De Burbure C, et al. Chlorinated pool attendance, atopy and the risk of asthma during childhood. Environ Health Perspect 2006; 114: 1567-1573.

22 Bernard A, Nickmilder M, Voisin C, et al. Impact of chlorinated swimming pool attendance on the respiratory health of adolescents. Pediatrics 2009; 124: 1110-1118.

23 Cotter A, Ryan CA. The pool chlorine hypothesis and asthma among boys. Ir Med J 2009; 102: 79-82.

24 Bernard A. Chlorination products: emerging links with allergic diseases. Curr Med Chem 2007; 14: 1771-1782.

25 Weaver WA, Li J, Wen Y, et al. Volatile disinfection by-product analysis from chlorinated indoor swimming pools. Water Res 2009; 43: 3308-33018.

26 Tatsumi T, Fliss H. Hypochlorous acid and chloramines increase endothelial permeability: possible involvement of cellular zinc. Am J Physiol 1994; 267: H1597-H1607.

27 Musch MW, Walsh-Reitz MM, Chang EB. Roles of ZO-1, occludin, and actin in oxidant-induced barrier disruption. Am I Physiol Gastrointest Liver Physiol 2006; 290: G222-G231.

28 Bernard A, Carbonnelle S, Michel O, et al. Lung hyperpermeability and asthma prevalence in schoolchildren: unexpected associations with the attendance of indoor chlorinated pools. Occup Environ Med 2003; 60: 385-394.

29 Sinclair RG, Jones EL, Gerba CP. Viruses in recreational water-borne disease outbreaks: a review. J Appl Microbiol 2009; 107: 1769-1780.

30 Moro MR, Bonville CA, Suryadevara M, et al. Clinical features, adenovirus types, and local production of inflammatory mediators in adenovirus infections. Pediatr Infect Dis J 2009; 28: 376-380.

31 Artieda J, Pineiro L, Gonzalez MC, et al. A swimming pool-related outbreak of pharyngoconjunctival fever in children due to adenovirus type 4, Gipuzkoa, Spain, 2008. Euro Surveill 2009; 14: pii=19125.

32 Ji Q, Zhang L, Wang L, et al. Pentoxifylline reduces indirect lung injury of fresh water drowning in canis. Clin Chim Acta 2006; 365: 221-229. 